\title{
Inclusion Problem with Polynomial Data in $R^{3}$
}

\author{
Ikhsan Maulidi
}

December 29, 2017

\begin{abstract}
In this paper, we studied inclusion problem with polynomials data in $R^{3}$. We provided a Theorem that guaranted the solution of this problem. Moreover, by using standard potential theory, we approached the solution by using Green's Theorem.
\end{abstract}

\section{Introduction}

In this paper, we studied incclusion problem with polynomials data in $R^{3}$. We provided a Theorem that guaranted the solution of this problem. Moreover, by using standard potential theory, we approached the solution by using Green's Theorem.

Let $x=\left(x_{1}, x_{2}, x_{3}\right) \in R^{3}$, thus for $\alpha=\left(\alpha_{1}, \alpha_{2}, \alpha_{3}\right)$ of non-negative integers we say $x^{\alpha}$ as monomial $x_{1}^{\alpha_{1}} x_{2}^{\alpha_{2}} x_{3}^{\alpha_{3}}$. The degree of $x^{\alpha}$ is $\alpha_{1}+\alpha_{2}+\alpha_{3}$.

A polynomial is said to be homogeneous of degree $m$ if it is a finite linear combination of monomial $x^{\alpha}$ of degree $m ; m=0,1,2, \ldots$.

Let $\mathcal{P}_{m}$ denotes the vector space of polynomials in $R^{3}$, homogeneous of degree $m$, and $\mathcal{H}_{m}$ is the subspace of harmonic polynomials of degree $m$, then we have property by Axler and Ramey following

$$
\mathcal{P}_{m}=\mathcal{H}_{m}+|x|^{2} \mathcal{P}_{m-2} .
$$

\section{Problem Formulation of Inclusion Problem}

Let $\Omega$ is a unit sphere in $R^{3}$, with the boundary $\partial_{\Omega}=\left\{x \in R^{3} \mid \quad x_{1}^{2}+x_{2}^{2}+x_{3}^{2}=\right.$ $1\}$. 
Let also

$$
D=\left\{x \in R^{3} \quad ; 0 \leq|x| \leq R<1\right\},
$$

then we have inclusion problem as follows

$$
\begin{gathered}
\nabla\left[\left(1+(k-1) \mathcal{X}_{D}\right) \nabla u\right]=0 \\
\left.u\right|_{\Omega}=p
\end{gathered}
$$

where $\mathcal{X}_{D}$ is a characteristic function and $p$ is an $n$-degree polynomial, $n \in \mathcal{P}_{n}$.

Let $u_{0}$ be the harmonic function on $\Gamma=\omega-D$ with boundary values $f$, that is

$$
\begin{gathered}
\Delta u_{o}=0 \quad \text { in } \Gamma . \\
\left.u_{o}\right|_{\Omega}=p .
\end{gathered}
$$

Let also $u_{i}$ is a function that satisfies

$$
\Delta u_{i}=0 \quad \text { in } \quad D
$$

the compatibility condition

$$
\left.u_{i}\right|_{\partial D}=\left.u_{o}\right|_{\partial D}
$$

and

$$
\frac{\partial u_{o}}{\partial n}=k \frac{\partial u_{i}}{\partial n}
$$

Then the problem (1) is equivalent problem with the problem (3) and (5) with the boundary problem (4) and (6).

The solution of (1) is

$$
u=u_{o} \mathcal{X}_{\Gamma}+u_{i} \mathcal{X}_{D}
$$

\section{Technical Lemmas}

This following Lemmas is needed to solve the Inclusion Problem:

Lemma 3.1 The Poincaré inequality Let $p$, so that $1 \leq p \leq \infty$ and $\Omega$ a subset with at least one bound. Then there exists a constant $C$, depending only on $\Omega$ and $p$, so that, for every function $u$ of the $W_{0}^{1, p}(\Omega)$ Sobolev space,

$$
\|u\|_{L^{p}(\Omega)} \leq C\|\nabla u\|_{L^{p}(\Omega)}
$$


Proof: See Lawrence Evans, 1998.

Lemma 2.2 The Lax-Milgram Theorem Let $\phi$ be a bounded coercive bilinier form on a Hilbert Space $H$, then for every bounded linear function $f$ on $H$, there exists a uniquex $\in$ $\in H$ such that

$$
f(x)=\phi\left(x, x_{f}\right)
$$

for all $x \in H$.

Proof : See Debnath L and Mikusinsky, 1990.

Lemma 2.3 The Green's Theorem Let $C$ be a positively oriented, piecewise smooth, simple closed curve in a plane, and let $D$ be the region boounded by $C$. If $L$ and $M$ are functions of $(x, y)$ defined on an open region containing $D$ and have continuous partial derivatives there, then

$$
\oint_{C}(L d x+M d y)=\iint_{D}\left(\frac{\partial M}{\partial x}-\frac{\partial L}{\partial y}\right) d x d y,
$$

where the path of integration along $C$ is counterclockwise.

(Steward J)

\section{The Main Theorem}

Here we provide the Theorem about the solution of the Inclusion problem. The Existence of this solution can be presented in the following Theorem:

Theorem 4.1 There is exist a unique solution, $u$, of problem (1) and (2).

Proof: For $p \in \mathcal{P}_{n}$, let $p$ denote an extension of the boundary value $p$ such that $p=0$ in a subdomain $\tilde{D}$ with $D \subset \tilde{D} \subset \Omega$.

Let $u^{0}=u-p$ so that $u^{0} \in(\mathcal{P})_{\ni}$ solves

$$
\int_{\Omega} a \nabla u^{0} \cdot \nabla v d x=-\int_{\Omega} a \nabla p \cdot \nabla v d x
$$

for all $v \in \mathcal{P}_{n}$, with $a=1+(k-1) \mathcal{X}_{D}$.

Since $a>0$, Poincares's inequality and the Lax-Milgram Theorem imply the existence of a unique solution $u^{0} \in \mathcal{P}_{n}$ (Gilbarg D and Trudinger). Therefore, we have $u=u^{0}+p$ is a unique solution. Standard potential theory can be used to obtaoin a representation of the solution (1) and (2), one that can actually be used as the basisof a numerical approximation scheme. 
Let $w$ be the harmonic function on $\Omega$ with boundary values $p \in \mathcal{P}_{n}$, that is

$$
\begin{array}{lll}
\Delta w=0 \quad \text { in } & \Omega . \\
w=p & 0 \mathrm{n} & \partial \Omega .
\end{array}
$$

Define $u^{0}=u-w$ in $\Omega$, so that $u^{0}$ is harmonic in $D$ and $\Omega-\bar{D}$.

$$
\begin{gathered}
\Delta u^{0}=0 \quad \text { in } \quad D . \\
\Delta u^{0}=0 \quad \text { in } \quad \Omega-\bar{D} .
\end{gathered}
$$

It is easy to check that $u^{0}$ vanishes on $\partial \Omega$, because

$$
\begin{gathered}
\left.u^{0}\right|_{\partial \Omega}=\left.(u-w)\right|_{\partial \Omega} \\
=p-p=0 .
\end{gathered}
$$

Let $G(x, y)$ denote the Green's function for the Laplacian in $\Omega$ subject to Dirichlet boundary condition. Then Green's Theorem yields the representations as folows:

for $x \in D$

$$
u^{0}(x)=\int_{\partial D}\left[\frac{u_{i}^{0}(y)}{\partial n} G(x, y)-u_{i}^{0}(y) \frac{\partial G(x, y)}{\partial n_{y}}\right] d S_{y},
$$

for $x \in \Omega-\bar{D}$

$$
u^{0}(x)=-\int_{\partial D}\left[\frac{u_{o}^{0}(y)}{\partial n} G(x, y)-u_{0}^{0}(y) \frac{\partial G(x, y)}{\partial n_{y}}\right] d S_{y},
$$

where $u_{i}^{0}=u_{i}-w$ and $u_{o}^{0}=u_{o}-w$.

\section{Acknowledgement}

I would like to thank A.D. Garnadi who gave me the problems as independent study. 


\section{References}

[1] Debnath L and Mikusinsky,P. 1990. Introduction to Hilbert space with applications.San Diego, CA : Academic Press.

[2] Evans, Lawrence C. 1998. Partial differential equations, Providence, RI: American Mathematical society, ISBN 0-8218-0772-2.

[3] S. Steward, James. Calculus (6th ed). Thompson, Brooks/Cole.

[4] Gibarg D and Trudinger N S. 1983. Elliptical Partial Differential Equations of Second Order. Berlin: Springer. 